Tropical Journal of Pharmaceutical Research March 2016; 15 (3): 535-545

ISSN: 1596-5996 (print); 1596-9827 (electronic)

(C) Pharmacotherapy Group, Faculty of Pharmacy, University of Benin, Benin City, 300001 Nigeria.

All rights reserved.

Available online at http://www.tjpr.org

Original Research Article

http://dx.doi.org/10.4314/tjpr.v15i3.15

\title{
Nobiletin Inhibits Expression of Inflammatory Mediators and Regulates JNK/ERK/p38 MAPK and PI3K/Akt Pathways in Human Osteoarthritic Chondrocytes
}

\author{
Jian-wei Liu', Chong-wei Hao ${ }^{2}$, Zhao-hui Wang ${ }^{1}$ and Dan Jin ${ }^{3 *}$ \\ ${ }^{1}$ Department II of Orthopedics, ${ }^{2}$ Department of PICU, Chenzhou No. 1 People's Hospital, Southern Medical University \\ (Guangzhou), Chenzhou, Hunan 423000, ${ }^{3}$ Department of Orthopedics and Traumatology, Nanfang Hospital, Southern Medical \\ University, Guangzhou, 510515, China
}

*For correspondence: Email: jdjindan43@hotmail.com; Tel: 0086-020-61641114; Fax: 0086-020-61641114

Received: 9 October 2015

Revised accepted: 5 February 2016

\begin{abstract}
Purpose: To investigate the anti-inflammatory effects of nobiletin on human osteoarthritic chondrocytes and also to explore possible related molecular events.

Methods: Isolated human osteoarthritic chondrocytes were stimulated with IL-1 $\beta$. The effect of nobiletin $(75,150$ or $300 \mu \mathrm{g})$ on chondrocyte viability was assessed. Furthermore, the effect on NO production was determined using Griess reagent while the levels of IL-6 and PGE2 were assessed by enzyme linked immunosorbent assay (ELISA). The influence of nobiletin on the expression of COX-2, iNOS and proteins of PI3/Akt, NF-KB and MAPK cascades were also assessed.

Results: Nobiletin $(75,150$ and $300 \mu g)$ significantly $(p<0.05)$ improved the viability of chondrocytes, and remarkably reduced the levels of NO, IL-6 and PGE2. The expression levels of COX-2 and iNOS both at mRNA and protein levels were strikingly reduced by nobiletin, in a dose-dependent way. In addition, nobiletin caused marked $(p<0.05)$ down-regulation of the NF-KB signalling pathway. IL-1 $1 \beta$ induced activation of PI3/Akt, and JNK, ERK and p38 MAPK cascades were significantly $(p<0.05)$ inhibited by nobiletin with $300 \mu \mathrm{g}$ dose exhibiting maximum effects.

Conclusion: Inflammatory cytokines are critically involved in the pathogenesis of OA. Significant suppression of cytokines and modulation of PI3/Akt and MAPK signalling cascades by nobiletin suggests its potent anti-inflammatory and anti-osteoarthritic effects.
\end{abstract}

Keywords: Chondrocytes, Inflammation, Mitogen-activated protein kinases, NF-KB, Nobiletin, Osteoarthritis

Tropical Journal of Pharmaceutical Research is indexed by Science Citation Index (SciSearch), Scopus, International Pharmaceutical Abstract, Chemical Abstracts, Embase, Index Copernicus, EBSCO, African Index Medicus, JournalSeek, Journal Citation Reports/Science Edition, Directory of Open Access Journals (DOAJ), African Journal Online, Bioline International, Open-J-Gate and Pharmacy Abstracts

\section{INTRODUCTION}

Osteoarthritis (OA) is a degenerative musculoskeletal disease characterized by progressive damage and erosion of articular cartilage, osteophyte formation and subchondral bone sclerosis eventually leading to pain and disability $[1,2]$. Chondrocytes of the articular cartilage play critical roles in pathogenesis of $O A$ by potentially expressing and as well responding to inflammatory cytokines [3]. These inflammatory cytokines perturb the homeostasis of metabolic processes in the joints [4].

$\mathrm{IL}-1 \beta$, a major cytokine in OA [5] induces the expression of matrix metalloproteinases (MMPs) such as collagenases (MMP-3 and -13). MMPs are involved in cartilage degradation that eventually leads to the release of matrix proteoglycans, a critical event in OA 
pathogenesis [6]. IL-1 $\beta$ induces activation of NF$\mathrm{KB}$ and MAPK signalling pathways in chondrocytes thereby enhancing the expression and release of NO and PGE2 via up-regulation of cyclooxygenase-2 (COX-2) and inducible nitric oxide synthase (iNOS) [7]. Studies have shown that NO up-regulates the production of MMPs and inflammatory cytokines in OA [8]. Moreover, studies have suggested activated phosphatidylinositol 3-kinase (PI3K) - protein kinase B (Akt) pathway to be associated in regulating chondrocyte survival [9]. Thus compounds that could inhibit the effects of IL-1 $\beta$ and modulate the NF-KB, PI3K/Akt and MAPK signalling pathways and prevent cartilage degradation are of great clinical value in the therapy of OA.

Recently, several researchers have focused on the effects of plant-derived compounds in curbing inflammatory responses in arthritis. Astragalin, a bioactive component from Rosa agrestis [10] and piperine derived from Piper nigrum exhibited antiinflammatory effects in OA model [11]. In our study, we investigated the effects of nobiletin in IL-1 $\beta$-induced OA. Nobiletin, a polymethoxylated flavone found in citrus peels has been reported to possess various biological properties as anticancer [12], anti-proliferative [13], antiinflammatory effects [14]. The present study was taken as an effort to explore the molecular events associated with the anti-inflammatory effects of nobiletin.

\section{EXPERIMENTAL}

\section{Chemicals and reagents}

Nobiletin was purchased from Sigma-Aldrich (St. Louis, MO, USA). Antibodies against p38, JNK, ERK, p-p38, p-ERK, p-JNK, p-NF-кB p65, p-ІкBa (Cell Signalling Technology Inc. Beverly, MA)

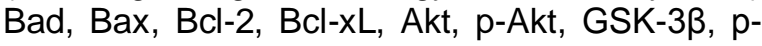
GSK-3 $\beta$, mTOR, PTEN, iNOS, TNF- $\alpha$ and COX2 (Santa Cruz, CA,USA) were used for western blotting. Griess Reagent was purchased from Beyotime Institute of Biotechnology (Shanghai, China). DMEM, FBS, collagenase II, penicillin and streptomycin were purchased from Gibco BRL (Grand Island, NY, USA). Recombinant human IL-1 $\beta$ was purchased from (R\&D systems. Minneapolis, MN, USA). All other chemicals used in the study were of analytical grade and were procured from Sigma-Aldrich (St. Loius, MO, USA) otherwise are specified.

\section{Chondrocyte isolation and cell culture}

Articular cartilage samples from 25 informed and consented patients (age: $57 \pm 13$ ) who were undergoing total knee replacement surgery were obtained. The study was approved by the institutional ethical committee. The articular cartilage was harvested from non-lesional areas and primary chondrocytes were isolated as described by Cheng et al [15].

In brief, the tissues were digested with trypsin $(0.25 \%)$ for $30 \mathrm{~min}$ and further digested using collagenase II $(0.2 \%)$ in DMEM for $6 \mathrm{~h}$ at $37^{\circ} \mathrm{C}$. The cells were suspended in DMEM with $10 \%$ fetal bovine serum (FBS), $100 \mathrm{U} / \mathrm{mL}$ of penicillin and $100 \mathrm{mg} / \mathrm{mL}$ of streptomycin and cultured at $\begin{array}{lllll}37 & { }^{\circ} \mathrm{C} \text { with } 5 & \% & \mathrm{CO}_{2} \text {. On reaching } 80 \%\end{array}$ confluence, the cells were pre-treated with nobiletin at 70,150 or $300 \mu \mathrm{g} / \mathrm{mL}$ for $6 \mathrm{~h}$ and were subsequently treated with $\mathrm{IL}-1 \beta(5 \mathrm{ng} / \mathrm{mL})$ for $24 \mathrm{~h}$.

\section{Cell viability assay}

The effect of nobiletin on the viability of chondrocytes was assessed by the MTT assay. The cells were seeded at a density of $6 \times 10^{3}$ in a 96-well plate and were cultured overnight, following which the cells were treated with various concentrations of nobiletin for $24 \mathrm{~h}$. The medium was discarded and $20 \mu \mathrm{L}$ MTT (5 $\mathrm{mg} / \mathrm{mL}$ ) was added to each well and incubated for $4 \mathrm{~h}$. After discarding the supernatant, $150 \mu \mathrm{L}$ of DMSO was added to solubilize the formazan crystals. The absorbance was measured at 570 $\mathrm{nm}$ using a micro-plate reader (Bio-Rad, Hercules, CA, USA).

\section{Annexin V/PI staining}

Cell apoptosis was measured by annexin V-FITC and $\mathrm{PI}$ staining. Chondrocyte cells that were exposed to nobiletin for $6 \mathrm{~h}$ and IL-1 $\beta$ for $24 \mathrm{~h}$ were seeded in a 24-well plate at a density of $2 \times$ $10^{5}$ cells per well. Apoptosis was detected using apoptosis detection kit (Santa Cruz Biotechnology, Santa Cruz, CA, USA). The cells were washed in PBS (phosphate buffered saline) and were then resuspended in binding buffer containing $100 \mu \mathrm{L}$ FITC-conjugated anti-annexin $\mathrm{V}$ antibodies. The cells were analyzed for fluorescence using a flow cytometer (FACS Calibur, BD Biosciences).

\section{Determination of IL-6 and PGE2}

Chondrocytes were pre-treated with nobiletin for $3 \mathrm{~h}$ and then stimulated with IL-1 $\beta$ for $24 \mathrm{~h}$. The concentrations of IL-6 and PGE2 in the culture medium were measured by ELISA ( $R$ \& $D$ Systems, Minneapoils, MN). 


\section{NO assay}

$\mathrm{NO}$ concentration in the culture medium of the chondrocytes treated with various concentrations of nobiletin and IL-1 $\beta$ as described above for IL-6 determination was assessed using Griess reagent as previously described by $\mathrm{Au}$ et al [16].

\section{RNA isolation and RT-PCR}

Real-time PCR was used to detect the expression of iNOS and COX-2. Total RNA was extracted using TriZol (Invitrogen) according to the manufacturer's instructions and quantified. RNA was quantified spectrophotometrically at $260 \mathrm{~nm}$ (HP 8452A Diode Array Spectrophotometer). First strand cDNA was synthesized using $0.3 \mathrm{mg}$ of the isolated RNA using DyNamoTM cDNA Synthesis Kit (Fermentas). Synthesized cDNA were amplified by PCR using the following primers: iNOS Forward - CCTTACGAGGCGAAGAAGGACAG, Reverse - CAGTTTGAGAGAGGAGGCTCCG; COX-2 Forward - GAGAGATGTATCCTCCCACA GTCA, Reverse - GACCAGGCACCAGACCA AAG. The relative expression of $\mathrm{COX}-2$ and iNOS were normalized with GADPH (primers Forward - TCTCCTCTGACTTCAACAGCGAC, Reverse - CCCTGTTGCTGTAGCCAAATT C). PCR was carried out as described by Ying et al [11].

\section{Western blot analysis}

Proteins extracted from chondrocytes treated with nobiletin $(70,150$ or $300 \mu \mathrm{g} / \mathrm{mL})$ and stimulated with IL-1 $\beta$ were subjected to western blotting. The cells were subjected to lysis buffer and the nuclear and cytoplasmic proteins were extracted from the cells using NE-PER Mammalian Protein Extraction Reagent (Thermo). Concentration of the isolated protein was determined using BCA protein assay kit (Bio-Rad Laboratories, USA). Equal amounts of protein samples $(40 \mu \mathrm{g})$ were separated on SDSpolyacrylamide gel (12\%) and transferred to PVDF membranes. The membranes were blocked with $5 \%$ non-fat dry milk and were incubated with primary antibodies overnight at 4 ${ }^{\circ} \mathrm{C}$ followed by incubation with peroxidaseconjugated secondary antibodies at room temperature for $60 \mathrm{~min}$. The immunoreactive bands were detected by enhanced chemiluminescence (GE Healthcare).

\section{Statistical analysis}

Data are presented as mean \pm SD ( $n=3$ or 6$)$. Statistical significance between the means of various experimental groups were assessed by one-way analysis of variance (ANOVA) followed by Duncan's Multiple Range Test as post-hoc analysis. Statistical difference at $p<0.05$ was considered significant.

\section{RESULTS}

\section{Cytotoxic effects of nobiletin}

Articular chondrocytes incubated with nobiletin (75, 150 or $300 \mu \mathrm{g} / \mathrm{mL}$ ) were subjected to MTT assay to assess if nobiletin had any cytotoxic effects. We observed that at all tested concentrations there was negligible effects on viability of chondrocytes (Fig. 1). The results suggest that nobilein exhibited no cytotoxic effects.

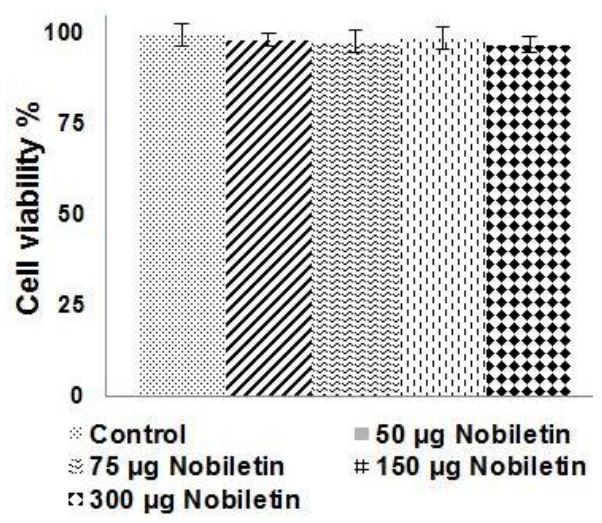

Figure 1: Influence of nobiletin on the cell viability of chondrocytes. Nobiletin had no cytotoxic effects on chondrocytes as determined by MTT assay; values are represented as mean $\pm S D, n=6$

\section{Nobiletin improves viability of chondrocytes exposed to IL-1 $\beta$}

Annexin V/PI staining and flow cytometry assay was employed to assess apoptosis of chondrocyte on exposure to IL-1 $\beta$. We observed apoptosis of chondrocytes stimulated with IL-1 $1 \beta$. However, pre-treatment with nobiletin interestingly caused a decrease $(p<0.05)$ in apoptosis, with $300 \mu \mathrm{g}$ concentration of nobiletin exhibiting maximum protective effects (Fig. 2). The low dose of $75 \mu \mathrm{g}$ though decreased apoptosis, the trend of reduction was significantly lesser as compared to higher concentrations of 150 and $300 \mu \mathrm{g}$. The results indicated that nobiletin effectively inhibited IL-1 $\beta$ - toxicity of chondrocytes.

Further to assess the influence of nobiletin on the expression of apoptotic pathway proteins, we analysed the expression of pro-apoptotic (Bax 
and $\mathrm{Bad}$ ) and anti-apoptotic (Bcl-2 and $\mathrm{Bcl}-\mathrm{xL})$ proteins. IL-1 $\beta$-stimulated chondrocytes expressed enhanced levels of pro-apoptotic proteins with decreased expression of $\mathrm{Bcl}-2$ and $\mathrm{Bcl}-\mathrm{xL}$ (Fig 2), suggesting raised apoptotic counts observed could have been due to raised levels of apoptotic protein expression. Interestingly, nobiletin caused significant upregulation in the levels of $\mathrm{Bcl}-2$ and $\mathrm{Bcl}-\mathrm{xL}$ while it down-regulated the expression of Bax and Bad. Thus, the results suggest the anti-apoptotic effects of nobiletin.

(a)

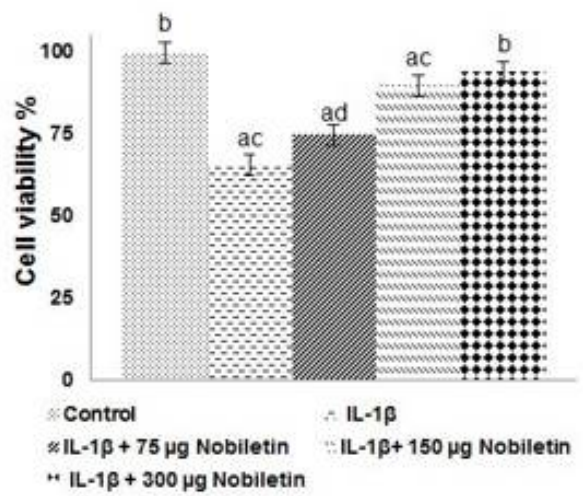

\section{Nobiletin inhibits IL-1 $\beta$-induced NO and PGE2 production}

NO and PGE2 levels in the culture medium of chondrocytes stimulated by $\mathrm{IL}-1 \beta$ were determined. Markedly $(p<0.05)$ enhanced levels of NO and PGE2 were observed. Multi-fold increases in the level of NO were seen. Moreover, increased levels of inflammatory cytokine,

(b)



Figure 2: Nobiletin inhibited IL-1 $\beta$ - induced apoptosis of chondrocytes. Nobiletin inhibited apoptosis of chondrocytes induced by IL-1 $\beta$ determined by Annexin $\mathrm{V}$ staining (a); and also modulated the expression of apoptosis pathway proteins (b; values are represented as mean $\pm S D(n=3)$. Key: $L 1=$ control; $L 2=I L-1 \beta$; L3 = IL-1 $\beta+75 \mu \mathrm{g}$ nobiletin; L4 = IL-1 $\beta+150 \mu \mathrm{g}$ nobiletin; L5=IL-1 $\beta+300 \mu \mathrm{g}$ nobiletin)

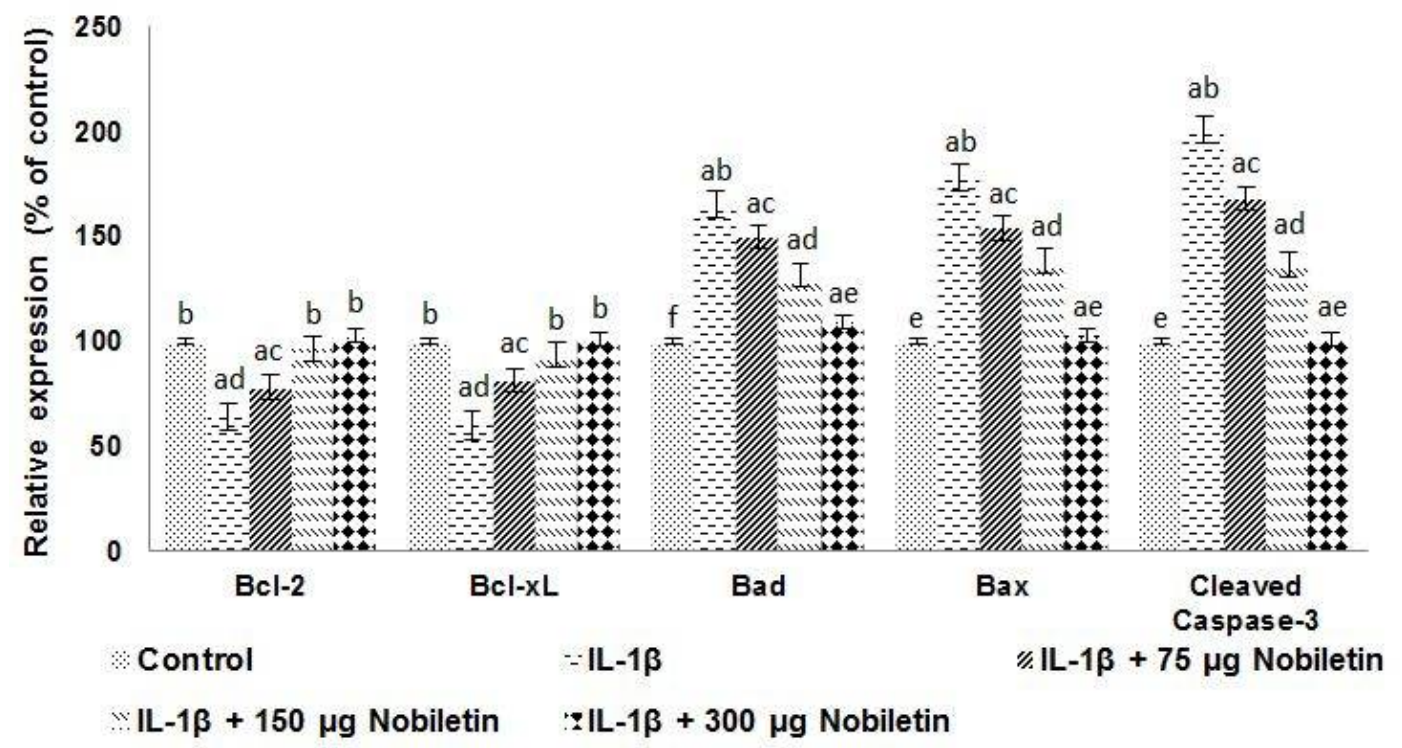

Figure 3 Nobiletin inhibited IL-1 $\beta$ - induced apoptosis of chondrocytes. Nobiletin modulated the expression of apoptosis pathway proteins); values are represented as mean $\pm \operatorname{SD}(n=3)$; a represents statistical significance at $p<0.05$ compared against control and b-f represents values within the same group that differ from each other at $p<0.05$ as determined by one-way ANOVA followed by DMRT analysis. Key: L1 = control; L2 = IL-1 $\beta$; L3 = IL$1 \beta+75 \mu \mathrm{g}$ nobiletin; L4 = IL-1 $\beta+150 \mu \mathrm{g}$ nobiletin; L5=IL-1 $\beta+300 \mu \mathrm{g}$ nobiletin) 
IL-6 were also observed upon IL-1 $\beta$ exposure (Fig. 3). Nobiletin caused significant $(p<0.05)$ decrease in the levels of NO, PGE2 and 1L-6. The results suggest the potent anti-inflammatory effects of nobiletin. The 150 and $300 \mu \mathrm{g}$ concentrations were more effective than $75 \mu \mathrm{g}$ nobiletin.

\section{Nobiletin suppressed iNOS and COX-2 expression}

$\mathrm{IL}-1 \beta$ is known to induce the expression of COX2 and iNOS [7], eventually leading to the production of PGE2 and NO. Here we investigated whether nobiletin influenced expression of iNOS and COX-2. In line with previous studies, we also observed markedly elevated mRNA iNOS and COX-2 expression (Fig. 4) and as well raised expression of iNOS and COX-2 at the protein levels (Fig. 6) in chondrocytes stimulated with IL-1 $\beta$. Nobiletin pre-treatment for $6 \mathrm{~h}$ caused a multi-fold decrease in the expression levels of both COX-2 and iNOS. The reductions in the expression at both the mRNA and protein levels were found to be dose-dependent, with $300 \mu \mathrm{g}$ concentration exerting maximum level of reduction. The reduction in the expression of $\mathrm{IL}-6, \mathrm{NO}$ and PGE2 were in line with down-regulated COX-2 and iNOS. The observations reveal that nobiletininduced decreased mRNA levels which could have contributed to reduced expressions of IL-6, $\mathrm{NO}$ and PGE2.

(a)

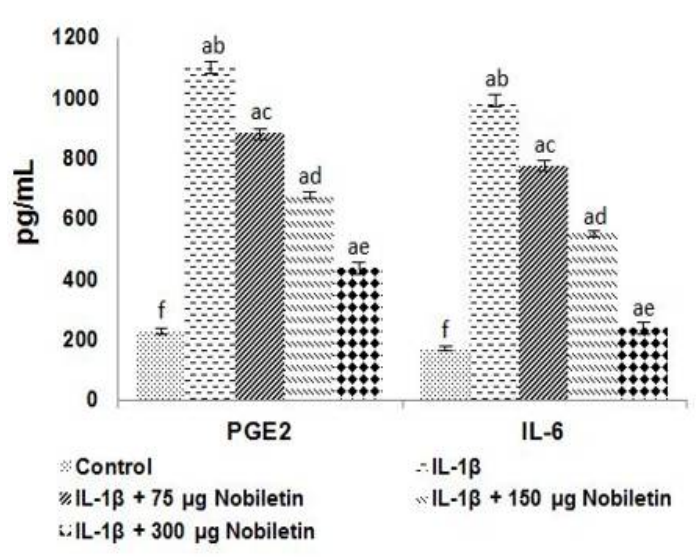

Effect of nobiletin on expression of MAPK cascade proteins

We assessed the expression of phosphorylated JNK, ERK and p38 MAPK. IL-1 $\beta$ stimulation caused a striking increase in the expressions of activated JNK, ERK and p38 MAPK (Fig. 5). Previous studies have shown enhanced expression of MAPKs under IL-1 $1 \beta$ stimulation [17]. However, nobiletin exposure resulted in significant down-regulation in the activation level of JNK, ERK and p38 MAPK. The phosphorylated JNK and p38 MAPK were highly reduced as compared to the $p$-ERK. The effects were similar at all the concentrations of nobiletin. The expression of ERK though reduced on exposure to nobiletin, the reduction was not significant as compared to JNK or p38MAPK.

\section{Nobiletin down-regulates NF-kB signalling}

$\mathrm{NF}-\mathrm{KB}$ is a transcription factor that critically regulates expression of inflammatory mediators. To investigate whether nobiletin exhibited antiinflammatory effects via modulating the NF-KB pathway, the expression of NF-KB pathway proteins were detected. IL-1 $\beta$ caused significantly up-regulated expression of $\mathrm{p}-\mathrm{NF}-\mathrm{KB}$ $\mathrm{p} 65, \mathrm{p}-\mathrm{I} \mathrm{KB} \alpha, \mathrm{IKB} \alpha$ and TNF- $\alpha$. Pre-treatment with nobiletin for $6 \mathrm{~h}$ markedly $(p<0.05)$ downregulated the expression in a dose-dependent manner (Fig. 6). This down-regulation of the pathway could have possibly contributed to the decreased expression of inflammatory mediators.

(b)

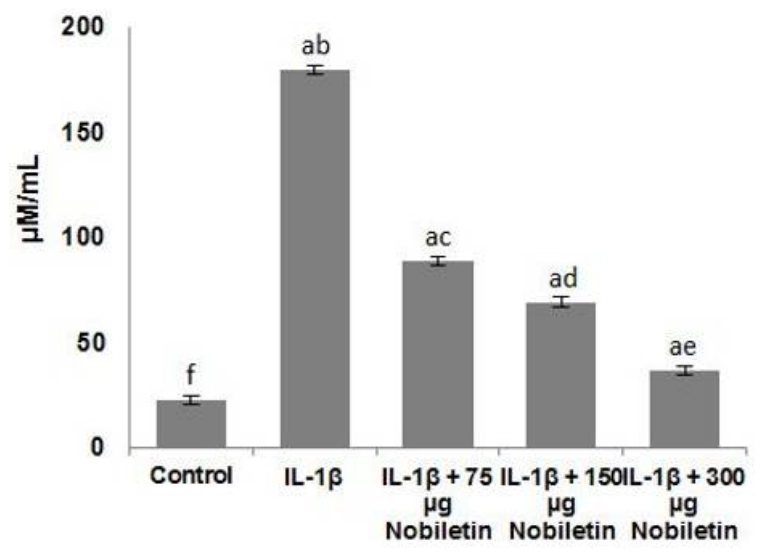

Figure 3: Influence of nobiletin on the levels of inflammatory mediators. Nobiletin effectively reduced levels of (a) PGE2 and IL-6 and (b) NO; values are represented as mean \pm SD $(n=6)$; a represents statistical significance at $p<0.05$ compared against control and b-f represents values within the same group that differ from each other at $p<0.05$ as determined by one-way ANOVA followed by DMRT analysis 
(a)

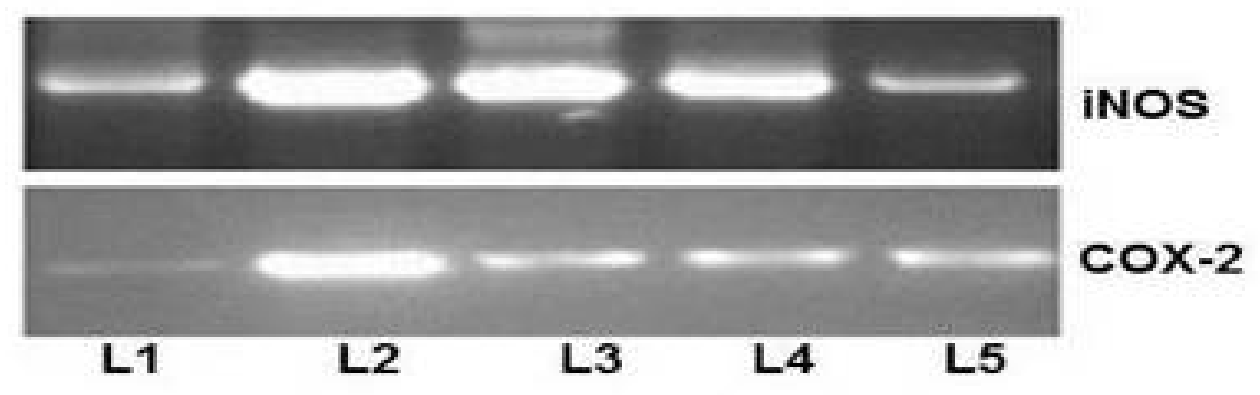

(b)

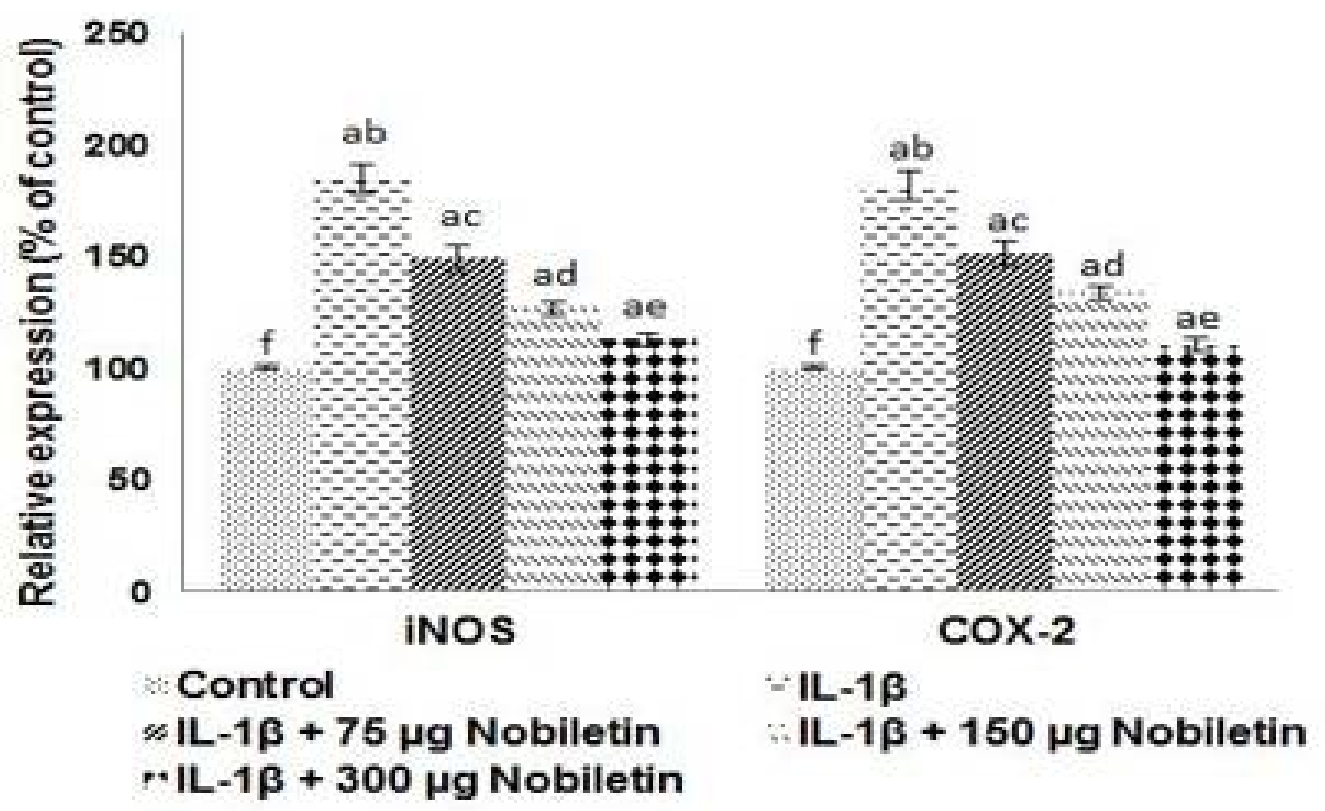

Figure 4: Effect of nobiletin on expressions of iNOS and COX-2. Nobiletin markedly reduced the expression of iNOS and COX-2 mRNA levels in IL-1 $\beta$-induced chondrocytes (a and b); values are represented as mean \pm SD $(\mathrm{n}=6)$; a represents statistical significance at $p<0.05$ compared against control and b-f represents values within the same group that differ from each other at $p<0.05$ as determined by one-way ANOVA followed by DMRT analysis. Key: $\mathrm{L} 1=$ control; $\mathrm{L} 2=\mathrm{IL}-1 \beta ; \mathrm{L} 3=\mathrm{IL}-1 \beta+75 \mu \mathrm{g}$ nobiletin; $\mathrm{L} 4=\mathrm{IL}-1 \beta+150 \mu \mathrm{g}$ nobiletin; $\mathrm{L} 5=\mathrm{IL}-1 \beta+$ $300 \mu \mathrm{g}$ nobiletin)

\section{Influence of nobiletin on the PI3K/Akt signalling pathway}

To investigate the anti-inflammatory mechanisms of nobiletin, we also assessed the expression of Akt pathway proteins. IL- $1 \beta$-induced chondrocytes exhibited elevated levels of Akt, phosphorylated Akt, phosphorylated GSK-3 $\beta$ and mTOR with decreased expression levels of
PTEN. Nobiletin at all the three analysed concentration was observed to significantly $(p<$ 0.05 ) inhibit the phosphorylation of Akt and GSK$3 \beta$ (Fig. 7). Furthermore, PTEN expression was found to be up-regulated by nobiletin in a dosedependent manner. Nobiletin thus effectively regulates the PI3K/Akt signalling pathway and this in part also contributed to the decreased expressions of NF-KB. 
(a)

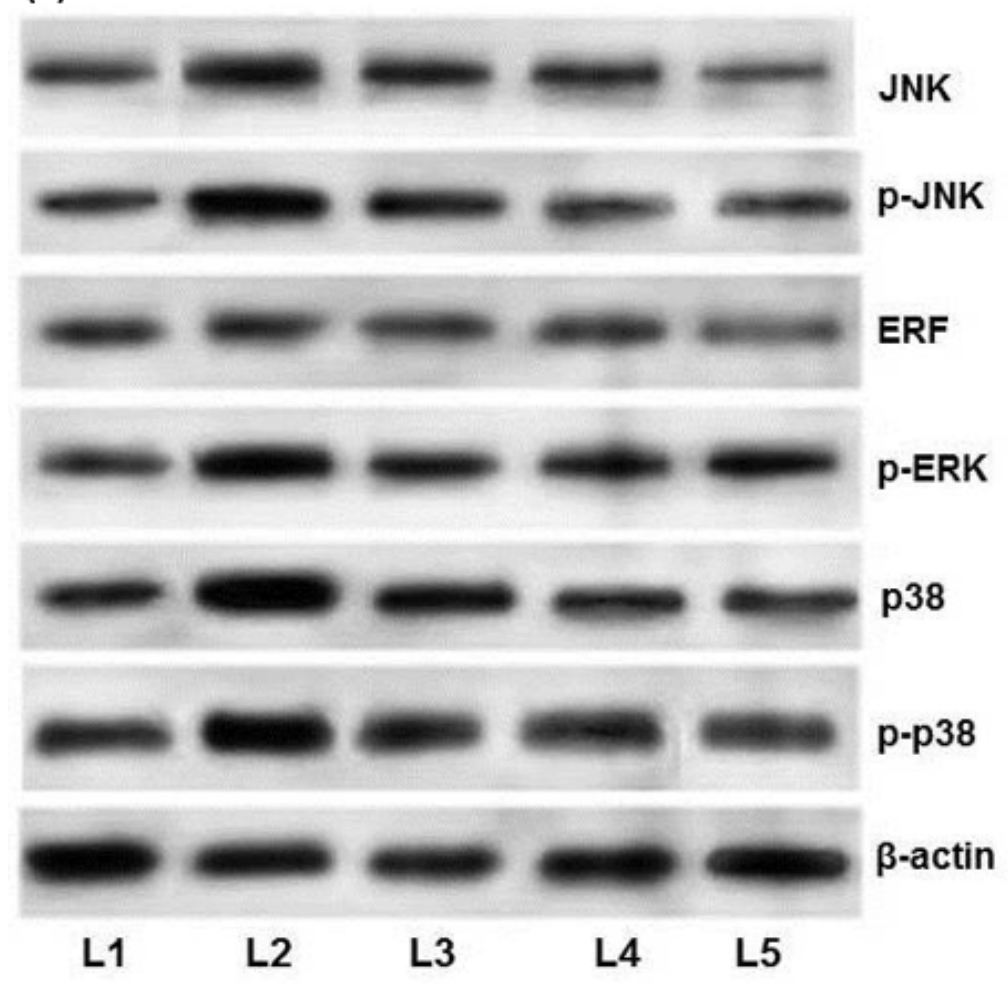

(b)

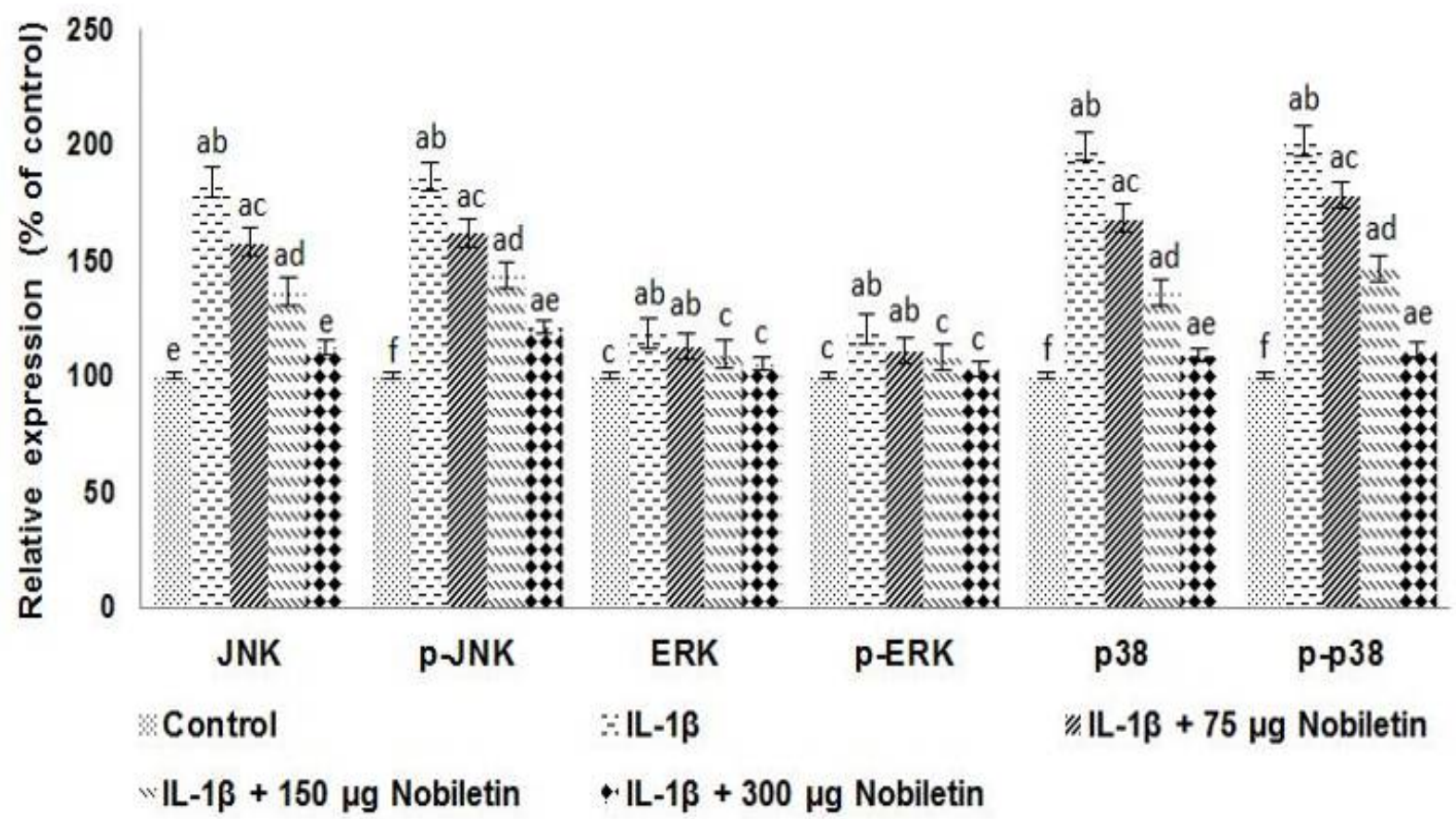

Figure 5: Nobiletin modulates MAPK pathway proteins. Nobiletin significantly down-regulated the MAPK pathways and inhibited activation and phosphorylation of JNK and p38 MAPK with slight noticeable changes on the expressions of ERK ( $a$ and $b)$; values are represented as mean \pm SD $(n=6)$; a represents statistical significance at $p<0.05$ compared against control and b-f represents values within the same group that differ from each other at $p<0.05$ as determined by one-way ANOVA followed by DMRT analysis. Key: $\mathrm{L} 1=$ control; L2 = IL$1 \beta ; \mathrm{L} 3=\mathrm{IL}-1 \beta+75 \mu \mathrm{g}$ nobiletin; $\mathrm{L} 4=\mathrm{IL}-1 \beta+150 \mu \mathrm{g}$ nobiletin; $\mathrm{L} 5=\mathrm{IL}-1 \beta+300 \mu \mathrm{g}$ nobiletin) 
(a)

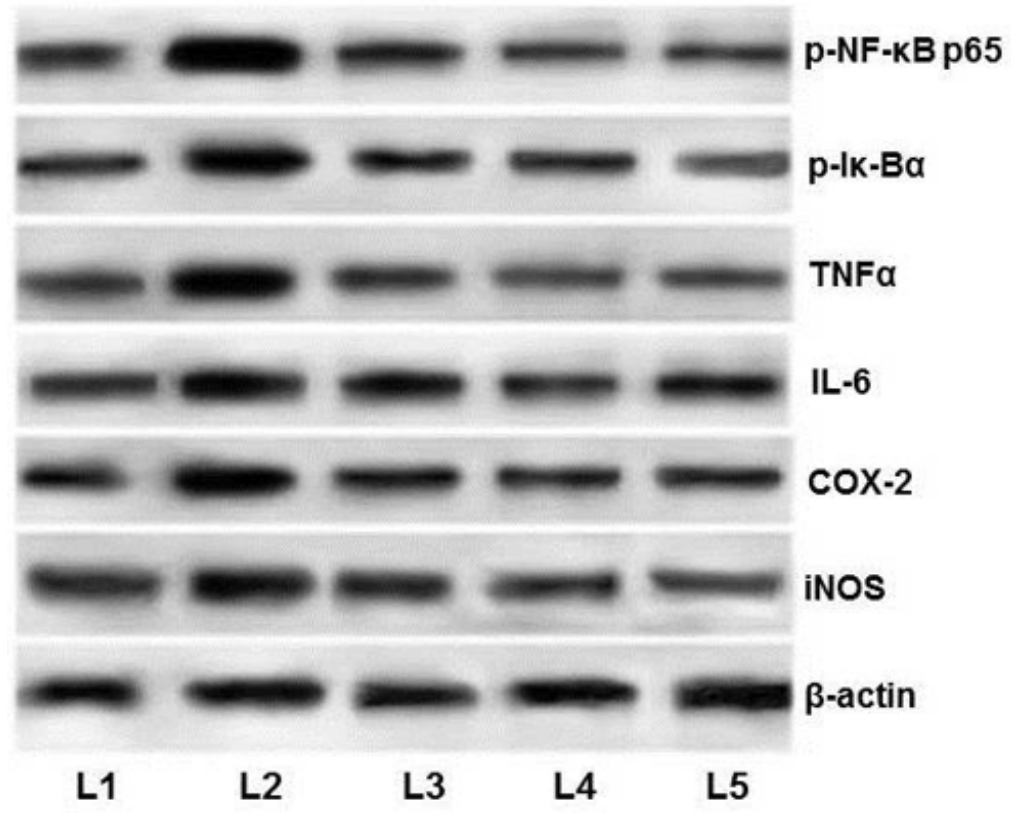

(b)

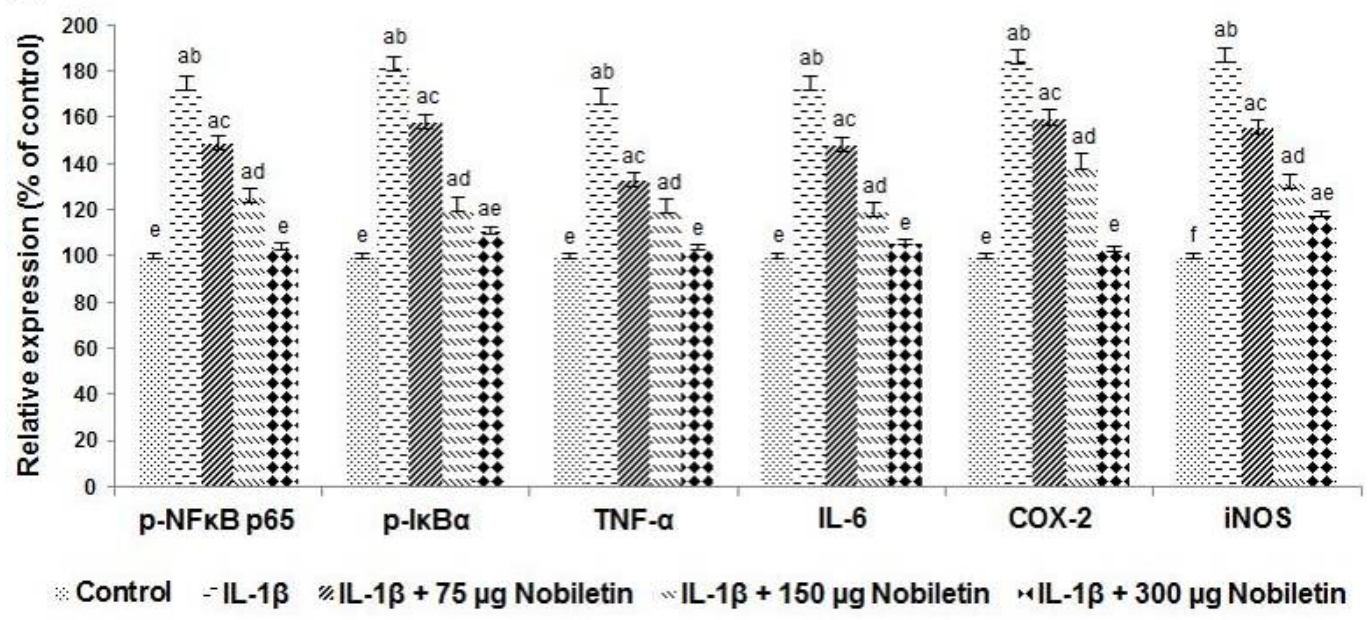

Figure 6: Nobiletin inhibiited NF-KB signalling pathway. IL-1 $\beta$-induced activation of the NF-KB signaling cascades and expressions of COX-2 and iNOS were effectively modulated and regulated by nobiletin ( $a$ and $b)$; values are represented as mean $\pm \operatorname{SD}(n=6)$; a represents statistical significance at $p<0.05$ compared against control and $b-f$ represents values within the same group that differ from each other at $p<0.05$ as determined by one-way ANOVA followed by DMRT analysis. Key: L1 = control; L2 = IL-1 $\beta$; L3=-IL-1 $\beta+75 \mu$ nobiletin; L4 = IL$1 \beta+150 \mu$ g nobiletin; $L 5=$ IL- $1 \beta+300 \mu$ g nobiletin)

\section{DISCUSSION}

Osteoarthritis $(\mathrm{OA})$ is a degenerative disease of the synovial joints that reduces quality of life [18]. Anti-inflammatory drugs prescribed for treating osteoarthritis are generally recommended for long term use. As individuals respond differently to drugs, chances of potential side effects remain as the major problem due to long term use of anti-inflammatory drugs. Recently, use of phytochemicals as anti-inflammatory agents is considered as an effective strategy to block inflammation in many inflammatory diseases such as OA [19]. In our study, we investigated the anti-inflammatory effects of nobiletin on IL$1 \beta$-induced inflammation in human osteoarthritic chondrocytes.

Role of inflammatory mediators NO and PGE2 is well known in the pathogenesis of $O A$ and increased production of $\mathrm{NO}$ and PGE2 have been reported in patients with OA [20]. IL-1B, an important inflammatory cytokine, is a major catabolic factor associated with $\mathrm{OA}$ exerting critical roles in cartilage degradation [21]. Elevated levels of IL-1 $\beta$ have been observed in synovial fluid in $O A$ [22]. IL-1 $\beta$ induces the expression of MMPs, such as MMP1, MMP3 and 
(a)

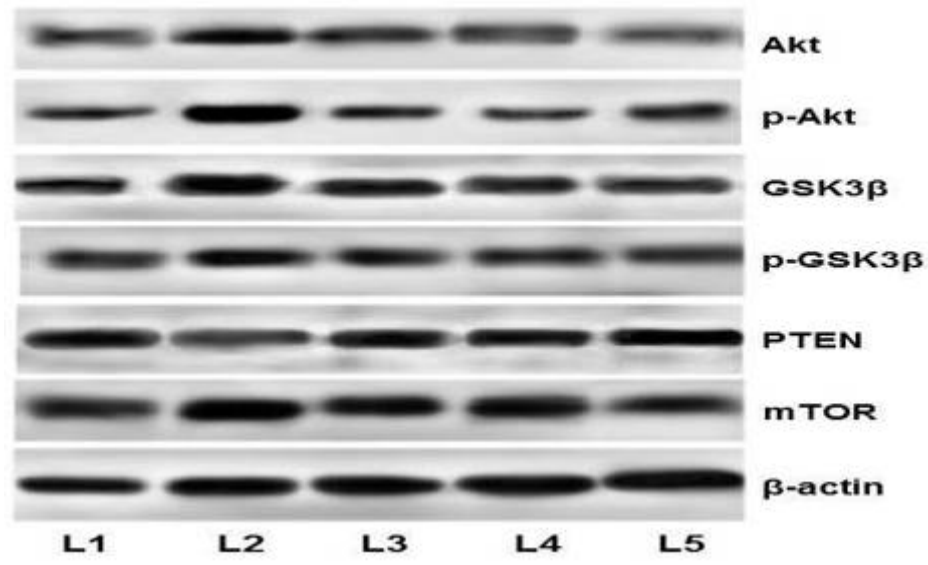

(b)
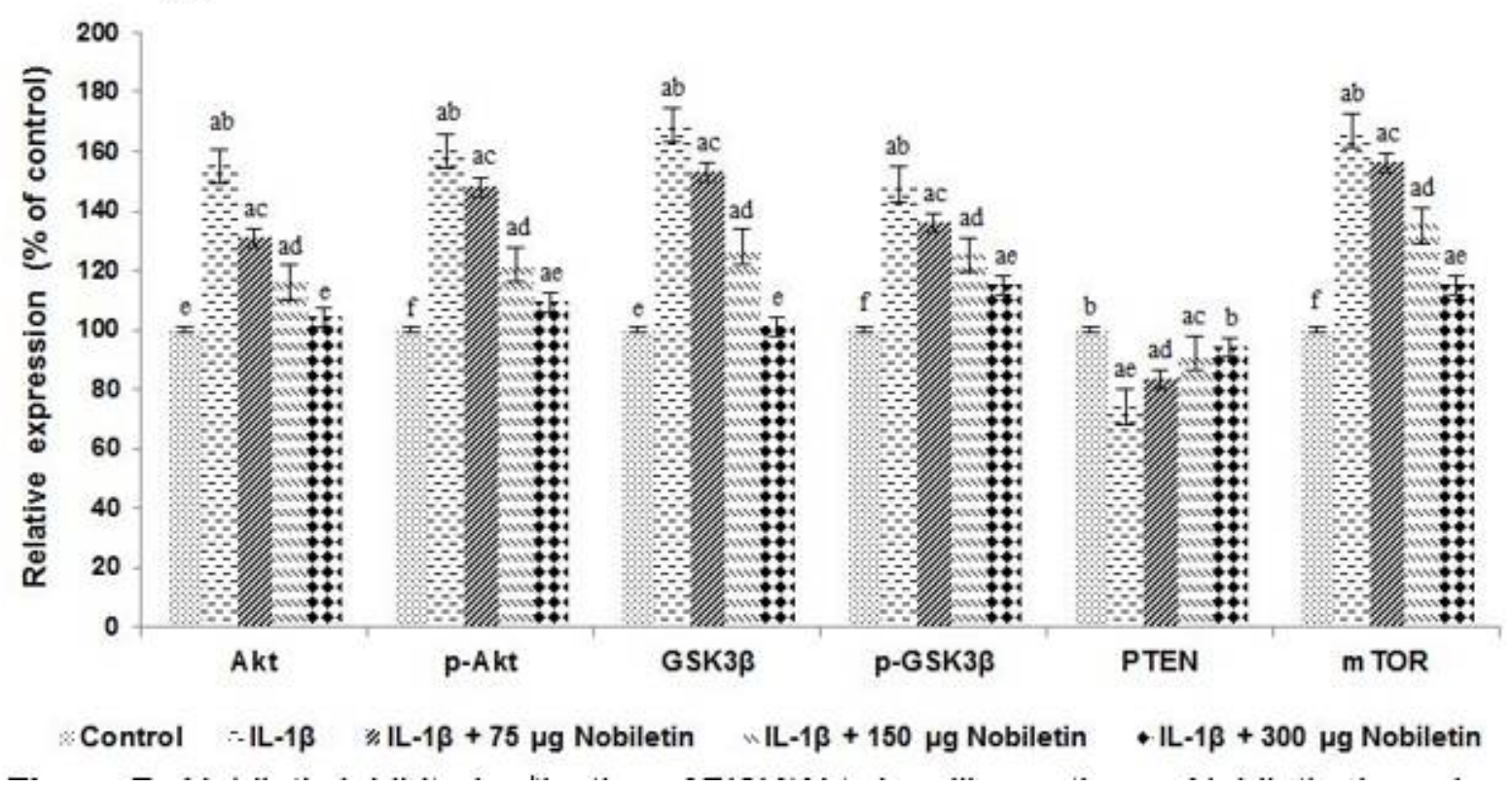

Figure 7: Nobiletin inhibited activation of PI3K/Akt signalling pathway. Nobiletin dose-dependently downregulated the expressions of proteins of the PI3K/Akt pathway, thereby inhibiting the pathway ( $a$ and $b$ ); values are represented as mean $\pm S D(n=3)$; a represents statistical significance at $p<0.05$ compared against control and $b-f$ represents values within the same group that differ from each other at $p<0.05$ as determined by one-way


$\mu \mathrm{g}$ nobiletin; $\mathrm{L} 5=\mathrm{IL}-1 \beta+300 \mu \mathrm{g}$ nobiletin

MMP13 that contribute to matrix degradation [23]. In addition, IL-1 $\beta$ induces the expression of iNOS and COX-2 and as well enhance the production of NO, PGE2 and IL-6 [11].

In the present study, we observed significant decrease in the levels of IL-6, NO and PGE2 as well as the expression of iNOS and COX-2 both at the mRNA and protein levels in response to pre-treatment with nobiletin. These observations suggest that nobiletin was able to effectively reduce the expression of inflammatory mediators, a hallmark event in OA therapy.
Nobiletin improved chondrocyte cell viability in a dose-dependent manner and had no cytotoxic effects on chondrocytes that were not exposed to IL-1 $\beta$. Nobiletin drastically altered the expression of apoptotic pathway proteins. Up-regulation of anti-apoptotic proteins with significant inhibition of pro-apoptotic proteins and caspase-3 in a dose-dependent way were also observed.

It is known that NF-KB and MAPK signalling pathways play critical roles in the regulation of inflammatory mediators. To further analyse the effects of nobiletin, we assessed the expression 
of NF $\mathrm{KB}$ and MAPK signalling pathway proteins. $\mathrm{NF}-\mathrm{KB}$ is one of the most important cytokineinduced transcription factors that critically regulate the expression of MMPs, COX-2, iNOS and other inflammatory cytokines [23]. Normally, $\mathrm{NF}-\mathrm{KB}$ is bound to IKB inhibitor proteins in the cytoplasm. IL-1 $\beta$ stimulation induces p65 phosphorylation and subsequent degradation of IKB that eventually leads to translocation of NFKB p65 into the nucleus that causes upregulation of inflammatory mediators [24]. In our study, nobiletin treatment caused remarkable down-regulation of phosphorylated NF-KB p65 and $\mathrm{IKB \alpha}$. The observed decreased expression of COX-2, iNOS and TNF- $\alpha$ suggest the inhibition of NF-KB signalling pathway by nobiletin that possibly could have also contributed to decreased levels of PGE2 and NO.

Previous studies have shown increased expression of MMPs, COX-2, and iNOS following stimulation of chondrocytes by IL-1 $\beta$ [25]. Thus, the results indicate that nobiletin exerts antiinflammatory effects via attenuation of NF-KB activity. Further, it has been reported that NF-kB signalling pathway mediates many of the biological effects of IL-1 $\beta$ on chondrocytes [26]. Thus inhibition of the pathway could be of immense clinical value in treatment of OA.

$\mathrm{PI} 3 \mathrm{~K}$ and Akt are the upstream molecules in NF$\mathrm{KB}$ pathway and have critical role in NF-KB activation [18]. To assess the effects of nobiletin on PI3K/Akt signalling, the expression of the pathway proteins was analysed. IL-1 $\beta$ caused multi-fold increased expression of phosphorylated Akt and GSK-3 $\beta$, indicating activation of the pathway. Nobiletin pre-treatment significantly inhibited the pathway as observed by decreased phosphorylation of Akt. The results suggest the possible involvement of PI3K/Akt cascades in nobiletin-induced inhibition of NF-KB activation.

MAPK pathways also exert critical effect the regulation of inflammatory cytokines production [27]. Binion et al. [28] reported that the activation of Akt further caused activation of MAPK pathways. Stimulating chondrocytes by IL- $1 \beta$ has been shown to induce phosphorylation and activation of ERK1/2, JNK, and p38 MAPKs [17]. In our study, enhanced expression of MAPK pathway proteins JNK, ERK and p38 were observed following IL-1 $\beta$ which is similar to the results of previous studies $[10,17]$. Nobiletin down-regulated the MAPK activation as evidenced by significant decrease in the expression of JNK, ERK and p38. Comparatively with the extent of JNK and p38 MAPK expression, ERK expression did not exhibit drastic changes, though some noticeable changes were seen. Nobiletin was able to effectively down-regulate MAPK pathways in IL$1 \beta$ stimulated chondrocytes which also in part could be responsible for reduced levels of inflammatory mediators observed.

\section{CONCLUSION}

Collectively, the observed results reveal the antinflammatory effects of nobiletin. Since nobiletin effectively modulates the critical pathways involved in $O A$ pathogenesis, it requires further investigation as a potent candidate in $\mathrm{OA}$ therapy.

\section{REFERENCES}

1. Ghosh $P$, Smith M. Osteoarthritis, genetic and molecular mechanisms. Biogerontology 2002; 3: 85-88.

2. Riddle DL, Stratford PW. Knee pain during daily tasks, knee osteoarthritis severity, and wide spread pain. Phys Ther 2014; 94: 490-498.

3. Daheshia M, Yao JQ. The interleukin 1beta pathway in the pathogenesis of osteoarthritis. J Rheumatol 2008; 35:2306-2312.

4. Kimura $M$, Kawahito $Y$, Obayashi $H$, Ohta $M$, Hara $H$, Adachi T, Tokunaga D, Hojo T, Hamaguchi M, Omoto A, Ishino $H$, Wada M, Kohno M, Tsubouchi Y, Yoshikawa T. Acritical role for allograft inflammatory factor-1 in the pathogenesis of rheumatoid arthritis. J Immunol 2007; 178: 3316-3322.

5. Honorati MC, Cattini L, Facchini A. IL-17, IL-1beta and TNF-alpha stimulate VEGF production by dedifferentiated chondrocytes. Osteoarthritis and Cartilage 2004; 12: 683-691.

6. Sinkov V, Cymet T. Osteoarthritis: understanding the pathophysiology, genetics, and treatments. J Natl Med Assoc 2003; 95: 475-482.

7. Chabane $N$, Zayed $N$, Afif $H$, Mfuna-Endam L, Benderdour M, Boileau C, Martel-Pelletier J, Pelletier JP, Duval N, Fahmi H. Histone deacetylase inhibitors suppress interleukin-1beta-induced nitric oxide and prostaglandin E2 production in human chondrocytes. Osteoarthritis Cartilage 2008; 16: 1267-1274.

8. Sasaki K, Hattori T, Fujisawa T, Takahashi K, Inoue $H$, Takigawa M. Nitric oxide mediates interleukin-1-induced gene expression of matrix metalloproteinases and basic fibroblast growth factor in cultured rabbit articular chondrocytes. J Biochem 1998; 123: 431-439.

9. Starkman BG, Cravero JD, Delcarlo M, Loeser RF. IGF-I stimulation of proteoglycan synthesis by chondrocytes requires activation of the $P$ I 3-kinase pathway but not ERK MAPK. Biochem J 2005; 389: 723-729.

10. Ma Z, Piao T, Wang $Y$, Liu J. Astragalin inhibits IL-1 $\beta$ induced inflammatory mediators production in human osteoarthritis chondrocyte by inhibiting NF-KB and 
MAPK activation. Int Immunopharmacol 2015; 25: 8387.

11. Ying $X$, Chen $X$, Cheng $S$, Shen $Y$, Peng $L, X u H Z$. Piperine inhibits IL-beta induced expression of inflammatory mediators in human osteoarthritis chondrocyte. Int Immunopharmacol 2013; 17: 293-299.

12. Suzuki R, Kohno H, Murakami A, Koshimizu K, Ohigashi $H$, Yano M, Tokuda $H$, Nishino H, Tanaka T. Citrus nobiletin inhibits azoxymethane-induced large bowel carcinogenesis in rats. BioFactors 2004; 22: 111-114.

13. Du $Q$, Chen $H$. The methoxyflavones in Citrus reticulate Blanco $\mathrm{CV}$. ponkan and their antiproliferative activity against cancer cells. Food Chemistry 2010; 119: 567572.

14. Murakami A, Shigemori T, Ohigashi $H$. Zingiberaceous and citrus constituents, 10-acetoxychavicol acetate, zerumbone, auraptene, and nobiletin, suppress lipopolysaccharide-induced cyclooxygenase-2 expression in RAW264.7 murine macrophages through different modes of action. J Nutrition 2005; 135: 2987 2992S.

15. Cheng AW, Stabler TV, Bolognesi M, Kraus VB. Selenomethionine inhibits IL-1beta inducible nitric oxide synthase (iNOS) and cyclooxygenase 2 (COX2) expression in primary human chondrocytes. Osteoarthr Res Soc 2011; 19: 118-125.

16. Au RY, Al-Talib TK, Au AY, Phan PV, Frondoza CG. Avocado soybean unsaponifiables (ASU) suppress TNF-alpha, IL-1beta, COX-2, iNOS gene expression, and prostaglandin E2 and nitric oxide production in articular chondrocytes and monocyte/macrophages. Osteoarthritis Cartilage 2007; 15: 1249-1255.

17. Akhtar N, Haqqi TM. Epigallocatechin-3-gallate suppresses the global interleukin-1beta-induced inflammatory response in human chondrocytes. Arthritis Res Ther 2011; 13: R93.

18. Reisch N, Engler A, Aeschlimann A, Simmen BR, Michel $B A$, Gay RE, Gay S, Sprott H. DREAM is reduced in synovial fibroblasts of patients with chronic arthritic pain: is it a suitable target for peripheral pain management? Arthritis Res Ther 2008; 10: R60

19. Kim KS, Oh da H, Choi HM, Bang JS, Ryu CJ, Kim JH, Yoo MC, Yang HI. Pyrrolidine dithiocarbamate, a NFkappaB inhibitor, upregulates MMP-1 and MMP-13 in IL1betastimulated rheumatoid arthritis fibroblast-like synoviocytes. Eur J Pharmacol 2009; 613: 167-175.
20. Koch B, Baum W, Burmester GR, Rohwer P, Reinke M, Zacher J, Kirchner H, Kalden JR. Prostaglandin E2, interleukin 1 and gamma interferon production of mononuclear cells of patients with inflammatory and degenerative joint diseases. Z Rheumatol 1989; 48 : 194-199.

21. Kobayashi M, Squires GR, Mousa A, Tanzer M, Zukor $D J$, Antoniou J, Feige U, Poole AR. Role of interleukin-1 and tumor necrosis factor alpha in matrix degradation of human osteoarthritic cartilage. Arthritis Rheum 2005; 52 : 128-135.

22. Wood DD, Ihrie EJ, Dinarello CA, Cohen PL. Isolation of an interleukin-1-like factor from human joint effusions. Arthritis Rheum 1983; 26: 975-983.

23. Klatt AR, Paul-Klausch B, Klinger G, Kuhn G, Renno JH, Banerjee M, Malchau G, Wielckens K. A critical role for collagen II in cartilage matrix degradation collagen II induces pro-inflammatory cytokines and MMPs in primary human chondrocytes. J Orthop Res 2009; 27:65-70.

24. Itthiarbha A, Phitak T, Sanyacharernkul S, Pothacharoen $P$, Pompimon $W$, Kongtawelert $P$. Polyoxypregnane glycoside from Dregea volubilis extract inhibits IL-1betainduced expression of matrix metallo proteinase via activation of NF kappa $B$ in human chondrocytes. In Vitro Cell Dev Biol Anim 2012; 48: 43-53.

25. Kang BJ, Ryu J, Lee CJ, Hwang SC. Luteolin inhibits the activity, secretion and gene expression of MMP-3 in cultured articular chondrocytes and production of MMP3 in the rat knee. Biomol Ther 2014; 22: 239-245.

26. Liacini A, Sylvester J, Li WQ, Zafarullah M. Inhibition of interleukin-1-stimulated MAP kinases, activating protein$1(A P-1)$ and nuclear factor $k B$ (NF-kB) transcription factors down-regulates matrix metalloproteinase gene expression in articular chondrocytes. Matrix Biol 2002; 21: 251-262.

27. Pawate $S$, Shen $Q$, Fan $F$, Bhat NR. Redox regulation of glial inflammatory response to lipopolysaccharide and interferon gamma. J Neurosci Res 2004; 77: 540-551.

28. Binion DG, Heidemann J, Li MS, Nelson VM, Otterson MF, Rafiee P. Vascular cell adhesion molecule1expressioninhumanintestinalmicro vascular endothelial cells is regulated by PI3-kinase/Akt/MAPK/NF-kappaB: inhibitory role of curcumin. Am J Physiol Gastrointest Liver Physiol 2009; 297: G259-G268. 